\title{
The effect of processing on the glucosinolate profile of mustard seed
}

\section{Katherine Cools, and Terry, L.A.*}

Plant Science Laboratory, Cranfield University, Bedfordshire, MK43 0AL, UK.

* Corresponding author. E-mail address: 1.a.terry@cranfield.ac.uk (L.A. Terry)

$$
\text { Tel.: }+44-7500-766-490
$$

Co-author e-mail address: k.cools@hortconsultancy.co.uk (K.Cools)

\begin{abstract}
Brassica juncea mustard seed are used to make mustard paste or condiment. Mustard seed contains glucosinolates which are converted to isothiocyanates following cell disruption by the enzyme, myrosinase. Isothiocyanates are sulphur-containing compounds which give a pungent flavour to the mustard condiment. Three mustard seed cultivars from two seasons were processed into Dijon- and wholegrain-style mustard and glucosinolates and isothiocyanates analysed. Canadian cv. Centennial tended to contain higher glucosinolates compared with the French cv. AZ147 and Ukrainian cv. Choraiva. Conversion of the mustard seed into a wholegrain condiment had less effect on total isothiocyanates and sinigrin content compared with the Dijon preparation. The Canadian mustard cultivars produced wholegrain-style mustard with higher total isothyocyantes and sinigrin compared with the French and Ukrainian cultivars. In summary, results herein suggest that Canadian mustard seed cvs. Centennial and and Forge, and wholegrain processing may result in a condiment with greater bioactive composition.
\end{abstract}

Keywords: Brassica juncea, condiment, cultivar, isothiocyanate, sinigrin. 


\section{INTRODUCTION}

Brassica juncea L. (syn. Sinapis juncea L.) is a hydrid between B. rapa and B. nigra giving it the characteristics of rapid growth from B. rapa and the mustard oil of B. nigra. There are two forms of B. juncea; the oilseed type and the vegetable type which is used for its edible leaves, stems and roots (Dixon, 2007). Mustard seed is used to make mustard condiment or paste. To make a smooth Dijon-style mustard condiment, the seeds are cracked (or milled into flour) and soaked in vinegar, then milled and sieved, whereas wholegrain mustard is simply cracked and soaked in vinegar. Mustard condiments are usually classed as mild or pungent; the mild mustard is often made with 'white' or yellow mustard (Sinapis alba or B. hirta) whereas the more pungent mustards are made with brown (B. juncea), oriental (blonde $B$. juncea) or black mustard (B. nigra) (Warseck, 1991). Dijon mustard from France should not contain yellow mustard seed, however in other countries the yellow and brown mustard seeds are often mixed to create a milder mustard condiment (Warseck, 1991).

In the genus, Brassica, over 90 different glucosinolates have been identified and these are classified into three sub groups; olefinic (sinigrin, gluconapin), methylsulfinylalkyl (glucoraphanin) and aryl (gluconasturtiin) (Kaushika and Agnihotri, 1999; Song et al., 2005). When enzymatically hydrolysed, glucosinolates yield isothiocyanates and give a pungent taste (Kaushika and Agnihotri, 1999). Each species of mustard seed has a different glucosinolate profile. The main glucosinolate of $B$. juncea is sinigrin which when hydrolysed by myrosinase yields allyl isothiocyanate whereas the main glucosinolate of $S$. alba is sinalbin which yields p-hydroxybenzyl isothiocyanate (Zasada and Ferris, 2004).

During the making of mustard condiment, it is possible that the glucosinolate content is affected through the lability of the isothiocyanates, resulting in reduced flavour. Nugrahedi et al. (2013) reviewed the effect of different food preparation techniques on Brassica 
vegetables. The aim of this study was to investigate whether glucosinolate and isothiocyanate concentrations could be maintained in different mustard condiments and whether specific cultivars of seed contained higher concentrations of bioactives over two seasons.

\section{MATERIALS AND METHODS}

\subsection{Plant Material}

Mustard seed (B. juncea) cvs. AZ147, Centennial, Chornaiva were acquired from France, Canada and Ukraine, respectively, and harvested in 2009. The Ukrainian cv. Chornaiva was not available from the 2010 harvest therefore this was replaced with Canadian cv. Forge. Mustard seed was supplied by Unilever R\&D Colworth (Beds., UK).

\subsection{Mustard preparation}

The experiment consisted of three cultivars harvested in 2009 and 2010. Each cultivar was analysed as unprocessed seed and after processing into Dijon- or wholegrain-style mustard. The spirit vinegar was supplied by Unilever R\&D Colworth and all other chemicals were bought from Sigma Aldrich (Dorset, UK) unless otherwise stated. Mustard seed was cracked using a pestle and immediately returned to liquid nitrogen to prevent myrosinase mediated conversion of glucosinolates to isothiocyanates. Seeds remained in a sealed bag while being cracked to minimise isothiocyanate loss. To produce $100 \mathrm{~mL}$ of mustard condiment, $29.26 \mathrm{~g}$ of cracked mustard was added to $55.66 \mathrm{~mL}$ of water, $8.90 \mathrm{~mL}$ spirit vinegar, $0.39 \mathrm{~g}$ citric acid, $5.57 \mathrm{~g}$ salt and $0.225 \mathrm{~g}$ potassium disulphide. The mixture was allowed to soak for $20-30 \mathrm{mins}$ at room temperature. To produce Dijon-style mustard, the mixture was then milled for 5 mins in a mechanised pestle and mortar and then passed through a sieve $(320 \mu \mathrm{m})$. To produce the wholegrain-style mustard, the milling and sieving steps were not carried out. The mustard 
preparations were allowed to mature for $24 \mathrm{~h}$ at room temperature and vortexed to remove air bubbles and thicken. The process was repeated 3 times per preparation.

\subsection{Isothiocyanates}

Isothiocyanates were extracted according to Cools and Terry (2012) and quantified according to Zhang et al., (1996). Mustard seed or mustard preparation was ground in liquid nitrogen and $200 \mathrm{mg}$ weighed into $7 \mathrm{~mL}$ bijou vials pre-cooled with liquid nitrogen. To each tube, $3 \mathrm{~mL}$ of boiling water was added and placed in a beaker of boiling water for a further 15 mins to denature the enzyme, myrosinase. The tubes were then cooled on ice and left in the fridge overnight to condense the volatiles before removing the vial lids. To the water extract, $500 \mu \mathrm{L}$ of dichloromethane (DCM) was added and mixed thoroughly. The DCM (100 $\mu \mathrm{L})$ was removed from the bottom of each tube and added to $1 \mathrm{~mL} 4 \mathrm{mM}$ benzene 1,2-dithiol and $1 \mathrm{~mL}$ $10 \mathrm{mM}$ potassium phosphate ( $\mathrm{pH} 8.5$ ), flushed with nitrogen and incubated for $2 \mathrm{~h}$ at $65^{\circ} \mathrm{C}$. In the place of the samples, $100 \mu \mathrm{L}$ of five known concentrations of allyl isothiocyanate; $2.5,5.0$, 7.5, 10 and $15 \mathrm{mM}$ were added to five separate reaction mixtures as known calibration standards. A blank mixture was also created where $100 \mu \mathrm{L}$ of pure DCM was added to a vial of reaction mixture. The reaction between the benzene 1,2-dithiol and the isothiocyanates yields the compound 1,3-benzodithiole-2-thione in a total isothiocyanate concentration dependent manner (Zhang et al., 1996).

After $2 \mathrm{~h}$ incubation, the concentration of 1,3-benzodithiole-2-thione was analysed by HPLC. Undiluted samples were injected $(20 \mu \mathrm{L})$ into an Agilent ZORBAX eclipse XDB-C18 column, $4.6 \times 150 \mathrm{~mm}, 5 \mu \mathrm{L}$ particle size (part no. 993967-902) with an Agilent ZORBAX eclipse XDB guard column, $1.0 \times 17 \mathrm{~mm}$ (part no. 5185-5921). The mobile phase consisted of $80 \%(\mathrm{v} / \mathrm{v})$ methanol at an isocratic flow rate of $1 \mathrm{~mL} \mathrm{~min}^{-1}$ and column temperature of $30^{\circ} \mathrm{C}$. The compounds were detected using an Agilent 1200 DA G1315B/G1365B photodiode array 
set to a wavelength of $365 \mathrm{~nm}$ and background wavelength of $600 \mathrm{~nm}$. The data was presented in Agilent ChemStation Rev. B.02.01 software. Concentration of 1,3-benzodithiole-2-thione was calculated against the reactions containing known quantities of allyl isothiocyanate. The standard chromatogram was compared against the blank chromatogram to identify the retention time of 1,3-benzodithiole-2-thione. The peak was eluted within 10 minutes.

\subsection{Sinigrin}

Sinigrin was extracted and quantified according to Cools and Terry (2012). Mustard seed was macerated in liquid nitrogen and weighed out into precooled vials. Mustard seed or preparations $(100 \mathrm{mg})$ were added to $3 \mathrm{~mL}$ boiling water then placed in boiling water for a further 15 mins to inactivate the myrosinase. Samples were then filtered using a $0.2 \mu \mathrm{m}$ MillexGV syringe driven filter unit. Samples were stored at $-40^{\circ} \mathrm{C}$ until further analysis.

Undiluted samples were injected $(10 \mu \mathrm{L})$ into an Agilent ZORBAX eclipse XDB-C18 column, 4.6 x 150mm, $5 \mu \mathrm{L}$ particle size (part no. 993967-902) with an Agilent ZORBAX eclipse XDB guard column, $1.0 \times 17 \mathrm{~mm}$ (part no. 5185-5921). The mobile phase consisted of $0.2 \mathrm{M}$ ammonium sulphate at an isocratic flow rate of $1.5 \mathrm{~mL} \mathrm{~min}^{-1}$ and column temperature of $25^{\circ} \mathrm{C}$. The compounds were detected using an Agilent $1200 \mathrm{DA}$ G1315B/G1365B photodiode array set to a wavelength of $229 \mathrm{~nm}$. The data was presented in Agilent ChemStation Rev. B.02.01 software. Concentration of sinigrin was calculated against an authentic standard.

\subsection{Total phenolics}

Mustard seed or preparation (150 mg) was mixed with $3 \mathrm{~mL}$ of 70:29.5:0.5 methanol:water:hydrochloric acid $(\mathrm{v} / \mathrm{v} / \mathrm{v})$ and vortexed to mix thoroughly. The vials were incubated at $37^{\circ} \mathrm{C}$ for $1.5 \mathrm{~h}$ in a shaking water bath vortexing every $15 \mathrm{mins}$ to prevent layering. 
Cooled samples were passed through a $0.2 \mu \mathrm{m}$ filter and stored at $-40^{\circ} \mathrm{C}$ until required (Giné Bordonaba and Terry, 2008). Briefly, $20 \mu \mathrm{L}$ of filtrate and $3.2 \mathrm{~mL}$ of distilled water were mixed with $200 \mu \mathrm{L}$ of Folin-Ciocalteu reagent followed by $600 \mu \mathrm{L}$ sodium carbonate (1.9M). After incubation at room temperature for $2 \mathrm{~h}$ in the dark, the absorbance was read at $760 \mathrm{~nm}$ using a Camspec M501 UV-Vis spectrophotometer (Camspec Ltd., Cambs., UK). Total phenolic content was calculated against a gallic acid standard curve and results expressed in mg of gallic acid equivalent (GAE) $\mathrm{g}^{-1}$ seed (Terry et al. (2007).

\subsection{Statistical analysis}

Analysis of variance (ANOVA) was conducted using Genstat for Windows Version 10.1.0.147 (VSN International Ltd., Herts., UK). Least significant differences (LSD; $P=0.05$ ) were calculated for each analysis. Significant differences are quoted at $P<0.05$ for all parameters unless otherwise stated. To identify relationships between the biochemical data sets, Pearson's Product Moment Correlation was adopted.

\section{RESULTS AND DISCUSSION}

The aim of this experiment was to investigate the impact of different cultivars and degree of processing on the fate of bioactives which impact flavour. Over two seasons, the Canadian cvs. Centennial and Forge were found to have higher sinigrin, total isothiocyanates and total phenolic content compared with other mustard seed cultivars and moreover, wholegrain-style mustard preparation preserved these compounds better than when prepared as Dijon mustard.

Two studies were conducted on B. juncea seed harvested in 2009 and 2010. In 2010, the Ukrainian seed was not available, therefore the Canadian cv. Forge from a 2010 harvest was analysed. The Canadian cv. Centennial had higher total isothiocyanates (Figure 1), 
sinigrin (Figure 2) and total phenolic content (Figure 3) in both years except for the isothiocyanate content in 2010 which was higher in Canadian cv. Forge. Higher isothiocyanate content in the Canadian cultivars may be due to higher substrate (sinigrin) and/or greater myrosinase activity. Myrosinase activity is inactivated by many processing techniques including boiling, steaming and other cooking techniques (Nugrahedi et al., 2015), yet mustard seed is not heated during Dijon-style and wholegrain condiment preparation. Since the Canadian cv. Forge was only analysed in 2010 , further research into this cultivar could prove beneficial. Although the literature on the health benefiting properties of isothiocyanates is conflicting, it is suggested that isothiocyanates supress tumour growth by producing reactive oxygen species or by inducing cycle arrest resulting in apoptosis (Gupta et al., 2014).

Pearson's Product Moment Correlation was carried out on the data as a whole and as separate harvest dates. There was no strong correlation between the data as a whole. There was a significant correlation between the total isothiocyanates and the total phenolic values ( $\mathrm{r}$ $=0.62$ ). In contrast, a negative correlation was found between the sinigrin and total phenolic values $(r=-0.67)$. Lower sinigrin would suggest higher isothiocyanate due to hydrolysis. Although the correlation data seems to suggest a link between isothiocyanate/sinigrin content and total phenolics; the 2-fold higher total isothiocyanate content found in cv. Forge in 2010 is not reflected in the total phenolic content in the same year. Correlation between data does not necessarily identify direct relationships i.e. that isothiocyanates have an antioxidant capacity, but rather that a mustard genotype with higher isothiocyanates may also contain higher phenolic compounds. Both isothiocyanates and phenolic compounds are defence related compounds; the most accepted theory is that the glucosinolate - myrosinase system exists for defence against herbivores/pathogens (Redovniković et al., 2008). The main phenolic compound of mustard seed flour (S. alba) was sinapic acid at $703.6 \mathrm{mg} 100 \mathrm{~g}^{-1}$ flour followed by hydroxybenzoic acid at $270.6 \mathrm{mg} 100 \mathrm{~g}^{-1}$ flour (Dabrowski and Sosulski, 1984). This said, 
S. alba is generally considered as a lower phenolic acid containing variety of mustard seed compared with $B$. juncea mustard seed; its paler colour has been attributed to its lower phenolic acid content. Similarly, mild mustard is often made with $S$. alba (B. hirta) seed as it contains lower concentrations of glucosinolates/isothiocyanates. Although the data on the glucosinolate/isothiocyanate and phenolic content of mustard seed and in particular mustard condiment is scarce, evidence herein suggests that less pungent varieties of mustard seed generally contain lower phenolic acid content. This may be due to varietal differences in breeding traits inherited/bred from the parent species; those associated with rapid growth from B. rapa and those associated with pungency from B. nigra.

Comparison between the different seed preparations showed that the seed alone had higher sinigrin (Figure 2) and lower isothiocyanates (Figure 1) compared to the mustard preparations, whereas the wholegrain-style mustard contained lower sinigrin and higher isothiocyanates (in 2009) compared with the seed. This suggests that during the mustard preparation, the enzyme myrosinase can break down sinigrin in the presence of high salinity and acidity from the mustard condiment additives (salt and spirit vinegar, respectively). Salt concentration during fermentation of cabbage (Brassica oleracea) was found to have no effect on glucosinolate content (Martinez-Villaluenga et al., 2009). In 2009, the isothiocyanate content of the three varieties was over double that of the Dijon-style mustard (Figure 1) suggesting not all isothiocyanates are lost during processing. Yet in 2010, the isothiocyanate content of the wholegrain was in line with the Dijon-style mustard suggesting volatile loss which may explain the poor correlation between the sinigrin data and isothiocyanate data. In the case of the Dijon-style mustard, the sinigrin content was especially low at an average of 0.46 and $0.2 \mathrm{mg} \mathrm{g}^{-1}$ seed compared with the wholegrain-style mustard in 2009 and 2010, respectively (Figure 2) suggesting substantial sinigrin breakdown during processing. The difference in isothiocyanate content of the wholegrain mustard between seasons illustrates the 
instability of this compound. There are many proposed mechanisms for glucosinolate reduction during processing (Nugrahedi et al., 2015). The additional maceration step included in the Dijon-style mustard preparation is likely to cause further cell lysis, diffusion of glucosinolates and myrosinase into the cell and possibly leaching. Thermal degradation is a common nonenzymatic mechanism for glucosinolate breakdown of processed Brassica vegetables, yet heat is not involved in mustard condiment preparation. In general, the wholegrain mustard contained higher sinigrin than the Dijon mustard suggesting that in terms of bioactive content and stability, the wholegrain mustard was superior.

In conclusion, the Canadian cvs. Centennial and Forge contained the highest sinigrin, total isothiocyanates and total phenolics. The higher concentrations of the above-mentioned compounds in the Canadian seed was higher in the wholegrain-style mustard. However, this was not the case for the Dijon-style mustard, possibly due to additional maceration step. Mustard seed varieties are often blended, therefore the Canadian cultivars, could be mixed with other varieties to maintain overall mustard appearance (Figure 4) and flavour.

\section{References}

Cools, K. and Terry, L.A. Terry. (2012) Comparative study between extraction techniques and column separation for the quantification of sinigrin and total isothiocyanates in mustard seed. Journal of Chromatography B 901, 115-118.

Dabrowski, K.J. and Sosulski, F.W. (1984) Composition of free and hydrolysable phenolic acids in defatted flours of ten oilseeds. Journal of Agricultural and Food Chemistry 32, 128130. 
Dixon, G.R. (2007) Origins and diversity of Brassica and its relatives In: Vegetable Brassicas and Related Crucifers. CABI, Oxfordshire, UK.

Giné Bordonaba, J. and Terry, L.A. (2008) Biochemical profiling and chemometric analysis of seventeen UK-grown black current cultivars. Journal of Agricultural and Food Chemistry 56, $7422-7430$.

Gupta, P., Kim, B., Kim, S-H. and Srivastava, S.K. (2014) Molecular targets of isothiocyanates in cancer: Recent advances. Molecular Nutrition \& Food Research 58, 1685-1707.

Kaushik, N. and Agnihotri, A. (1999) High-performance liquid chromatography method for separation and quantification of intact glucosinolates. Chromatographia 49, 281-284.

Martinez-Villaluenga, C., Peñas, E., Frias, J., Ciska, E., Honke, J., Piskula, M.K., Kozlowska, H. and Vidal-Valverde, C. (2009) Influence of fermentation conditions on glucosinolates, ascorbigen, and ascorbic acid content in white cabbage (Brassica oleracea var. Capitata cv. Taler) cultivated in different seasons. Journal of Food Science 74, C62-C67.

Nugrahedi, P.Y., Verkerk, R., Widianarko, B. and Dekker, M. (2015) A mechanistic perspective on process-induced changes in glucosinolates content in Brassica vegetables: A review. Critical Reviews in Food Science and Nutrition 55, 823-838.

Redovniković, I.R., Glivetić, T., Delonga, K. and Vorkapić-Furač, J. (2008) Glucosinolates and their potential role in plants. Periodicum Biologorum 110, 297-309.

Song, L., Morrison, J.J., Botting, N.P. and Thornalley, P.J. (2005) Analysis of glucosinolates, isothiocyanates, and amine degradation products in vegetable extracts and blood plasma by LC-MS/MS. Analytical Biochemistry 347, 234-243. 
Terry, L.A., Chope, G.A. and Giné Bordobaba, J. (2007) Effect of water deficit irrigation and inoculation with Botrytis cinerea on strawberry (Fragaria $\mathrm{x}$ ananassa) fruit quality. Journal of Agricultural and Food Chemistry 55, 10812-10819.

Warseck, M.R. (1991) Food Product containing non-pungent Dijon mustard flavouring and a process for making mustard paste. United States Patent. Patent number: 5,023,105.

Zasada, I.A. and Ferris, H. (2004) Nematode suppression with brassicaceous amendments: application based upon glucosinolate profiles. Soil Biology and Biochemistry 36, 1017-1024.

Zhang, Y., Wade, K.L., Prestera, T., and Talalay, P. (1996) Quantitative determination of isothiocyanates, dithiocarbamates, carbon disulfide, and related thiocarbonyl compounds by cyclocondensation with 1,2-benzenedithiol. Analytical Biochemistry 239, 160-167.

Figure captions

Figure 1 Total isothiocyanate concentration ( $\mu \mathrm{mol} \mathrm{g} \mathrm{g}^{-1}$ seed) of Dijon, wholegrain and mustard seed prepared using French AZ147, Canadian Centennial and Ukrainian Choraiva harvested in 2009 (top) and French AZ147, Canadian Centennial and Canadian Forge harvested in 2010 (bottom). LSD bar $(\mathrm{P}<0.05)$.

Figure 2 Sinigrin concentration $\left(\mathrm{mg} \mathrm{g}^{-1}\right.$ seed) of Dijon, wholegrain and mustard seed prepared using French AZ147, Canadian Centennial and Ukrainian Choraiva harvested in 2009 (top) and French AZ147, Canadian Centennial and Canadian Forge harvested in 2010 (bottom). LSD $\operatorname{bar}(\mathrm{P}<0.05)$.

Figure 3 Total phenolic concentration (mg GAE g ${ }^{-1}$ seed) of Dijon, wholegrain and mustard seed prepared using French AZ147, Canadian Centennial and Ukrainian Choraiva harvested in 
2009 (top) and French AZ147, Canadian Centennial and Canadian Forge harvested in 2010 (bottom). LSD bar $(\mathrm{P}<0.05)$.

Figure 4 Mustard preparations of Dijon (top) and wholegrain (bottom) mustard made using different mustard seed cultivars; Canadian Centennial, Canadian Forge and French AZ147. 


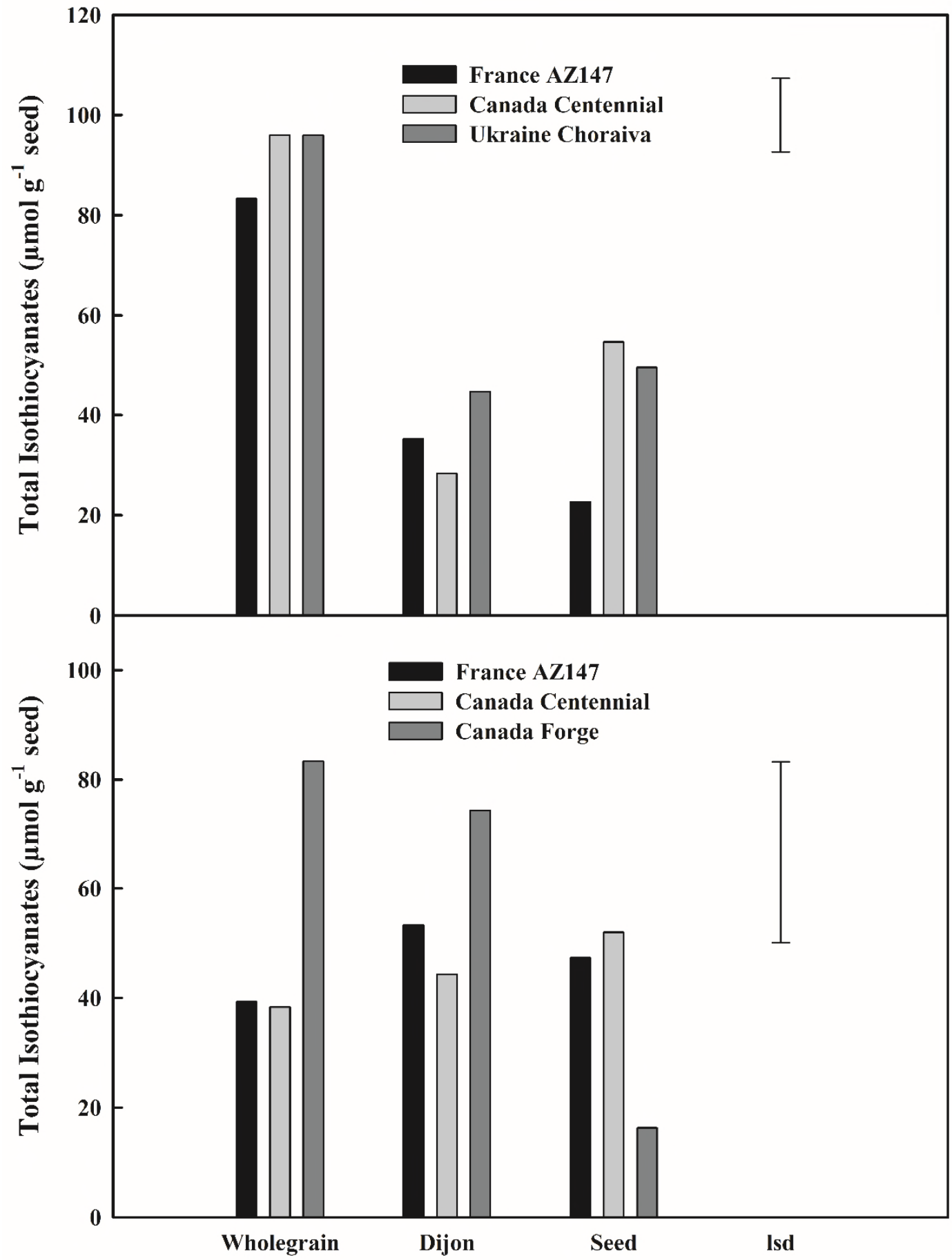




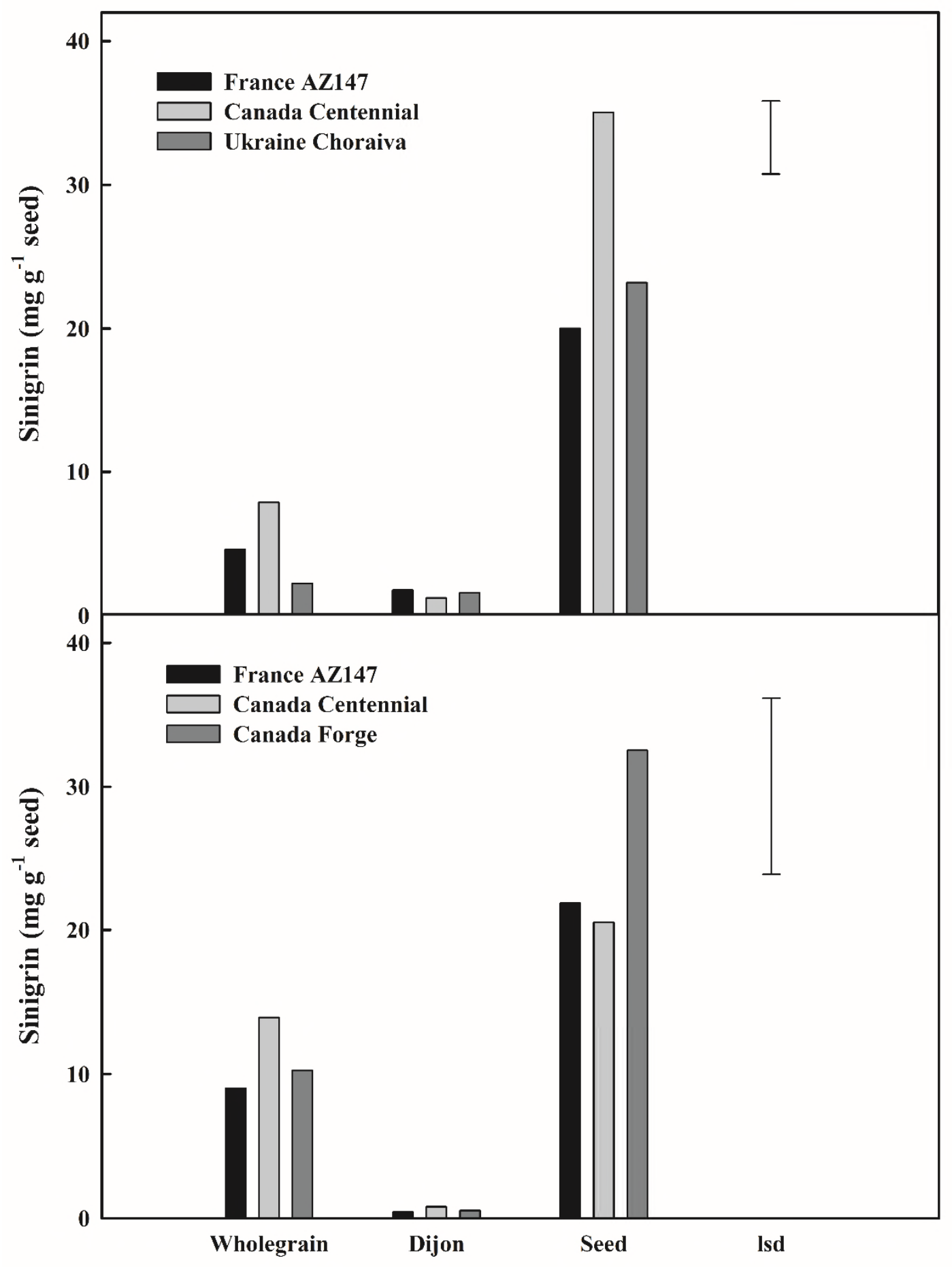




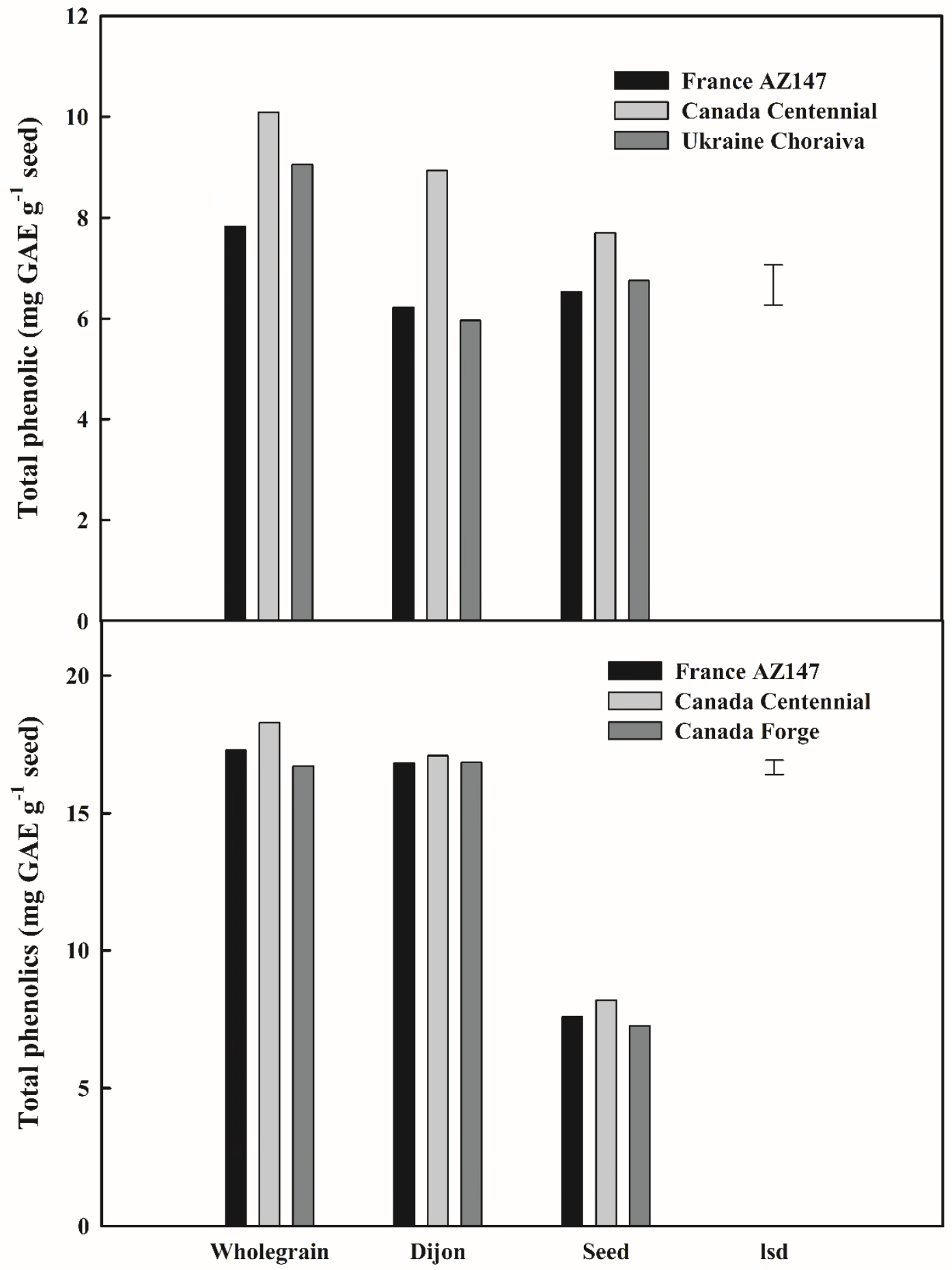




\section{Dijon}

Wholegrain
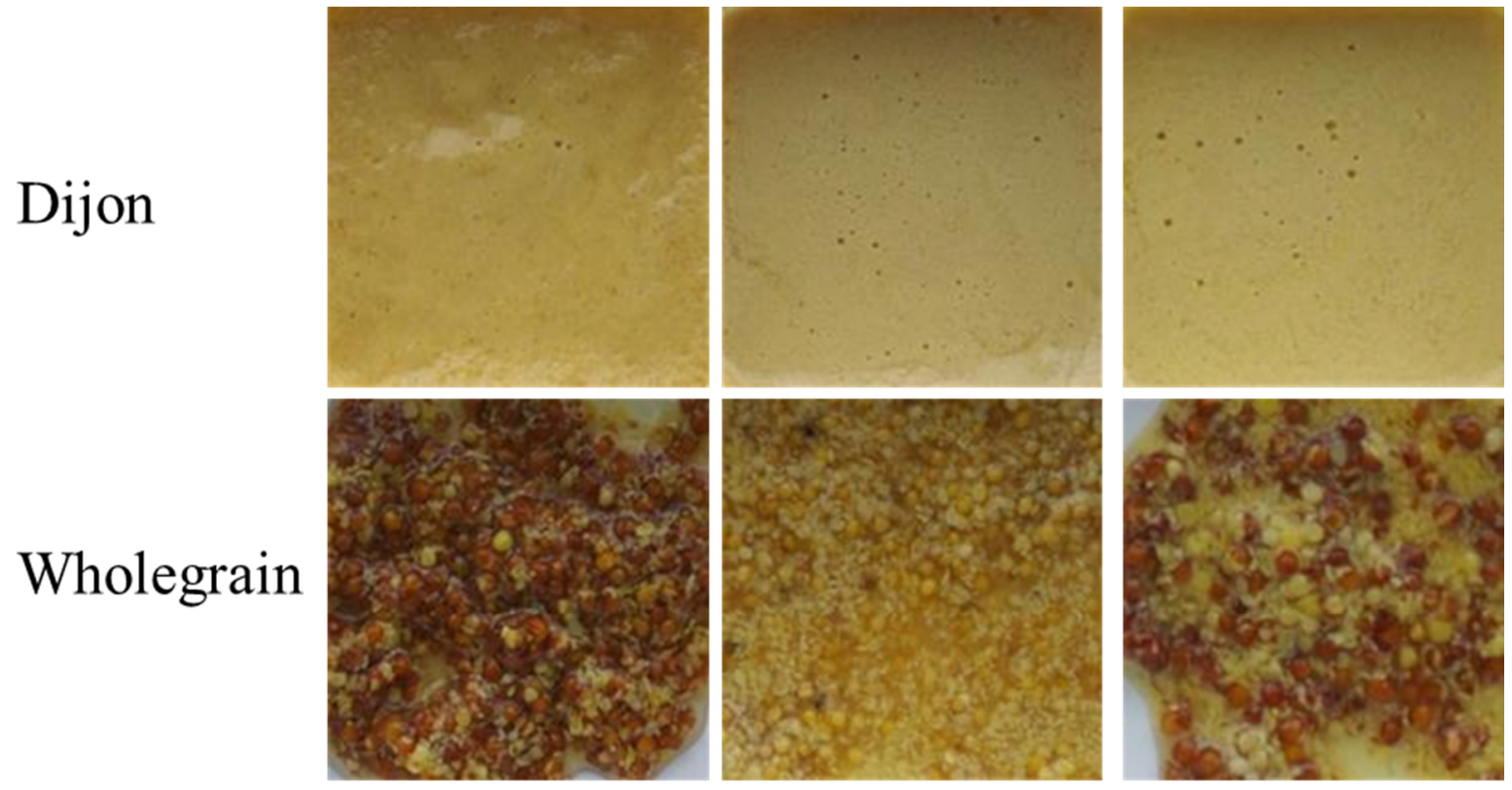

Centennial

Forge

AZ147 